\title{
Outcome of Dual 90-90 Plating in Intercondylar Fractures of Distal Humerus in Adults
}

\author{
Shrestha $D^{1}$, Shrestha $M^{2}$
}

\begin{abstract}
Background: Intercondylar fracture of distal humerus are very difficult to manage. It needs proper anatomic reconstruction and rigid fixation. Aims and Objectives of study: Evaluation of outcome of dual bicolumnar 90-90 plating of AO type 13C fractures of distal humerus in adult. Material and method: In a prospective hospital based study on 16 patients with a mean age of 33.37 years \pm SD 8.30, who presented with intercondylar fracture of distal humerus were studied between July 2015 to December 2017. All patients were graded according to AO classification. Out of 16 patients 5 were in 13C1, 6 were in $13 \mathrm{C} 2$ and 5 were in $13 \mathrm{C} 3$ subgroup. All patients underwent open reduction through Chevron olecranon osteotomy and fixation was done with dual plating in 90-90 fashion i.e.; 3.5 reconstruction plate posteriorly and $1 / 3$ tubular plate from medial border. We used the Mayo elbow performance score system for evaluating our results. Results: The mean follow up period was 9.38 months. All fractures healed uneventfully. Two of five patients with $13 \mathrm{C} 3$ fractures and two of six patients with $13 \mathrm{C} 2$ fractures has flexion deformity of less than $30^{*}$. Average range of motion was 95*(range 60*-130*). The Mayo elbow performance score was excellent in 7, good in 7 and fair in 2 patients. Conclusion: Dual 90-90 plating technique through Chevron olecranon osteotomy is a good method for intercondylar reconstruction with rigid fixation like $\mathrm{AO} 13 \mathrm{C}$ fractures.
\end{abstract}

Key words: Chevron olecranon osteototomy, dual plating, intercondylar fracture, mayo elbow performance score

\section{INTRODUCTION}

Intercondylar fracture of distal humerus in adult is one of the most difficult fractures to treat due to its complex structure ${ }^{1}$. Proper anatomic reconstruction and rigid fixation are the basic prerequisites for good clinical outcome and minimizes the complication such as nonunion, malunion, decreased range of motion and heterotrophic ossification. The incidences of distal humeral fractures in adult represent 5.7 per 100,000 persons per year. They constitute $30 \%$ of humerus fractures and $2 \%$ of all the fractures $s^{2,3}$. Open reduction and internal fixation with plates are to be applied on both the columns in AO type $13 \mathrm{C}$ fractures; however there is no standard protocol from which side the plates were to be placed ${ }^{4}$.

\section{MATERIAL AND METHODS}

Ethical permission for the study was obtained from the Institutional review committee of Nepalgunj Medical College Teaching Hospital (NGMCTH), Kohalpur and from the patient party. This was prospective hospital based study carried out from July 2015-December 2017.The study subjects were total 16 adults ranging from $24-48$ years.

1. Dr. Dinesh Shrestha

2. Dr. Merina Shrestha

Address for correspondence:

Dr. Dinesh Shrestha

Department of Orthopedics

Nepalgunj Medical College \& Teaching Hospital

Kohalpur, Banke

Email: dr_dineshstha@yahoo.com
We have treated all patients with stable or unstable and communited closed intercondylar distal humerus fractures. All patients were followed up for 1 year. Among them 10 patients were male and 6 were female.

After clinical assessment of the fracture carried out by the orthopaedic surgeon, three views of $x$-ray of elbow were done including antero-posterior, oblique and lateral, followed by CT scan with three dimensional reconstruction images which illustrated fracture level, area and degree of comminution, articular incongruity and column involvement ${ }^{5}$. Fractures were graded according to AO classification. Out of 16 patients 5 were in $13 C 1$ subgroup, 6 were in $13 C 2$ and 5 were in $13 C 3$ subgroup. Among them 3 patient developed impending compartment syndrome and 1 had subdural haematoma. The patients were operated as soon as they were fit. Patients with established compartment syndrome, open fractures, previously malunited fractures and neurovascular injuries were excluded from the study.

\section{Surgical Technique}

The patients were placed in lateral decubitus position. Tourniquet was applied and distal humerus was approached through Chevron osteotomy of olecranon. Ulnar nerve was isolated and protected. Dual plating were done in all cases. We used $3.5 \mathrm{~mm}$ reconstruction plate on lateral column posteriorly and $3.5 \mathrm{~mm}, 1 / 3$ tubular plate on medial column medially in all the patients. Intraoperatively range of motion and stability of fracture fixation was assessed by doing flexion, extension, pronation and supination of elbow. Chevron olecranon osteotomy was fixed with $\mathrm{K}$-wires and tension band wiring. 

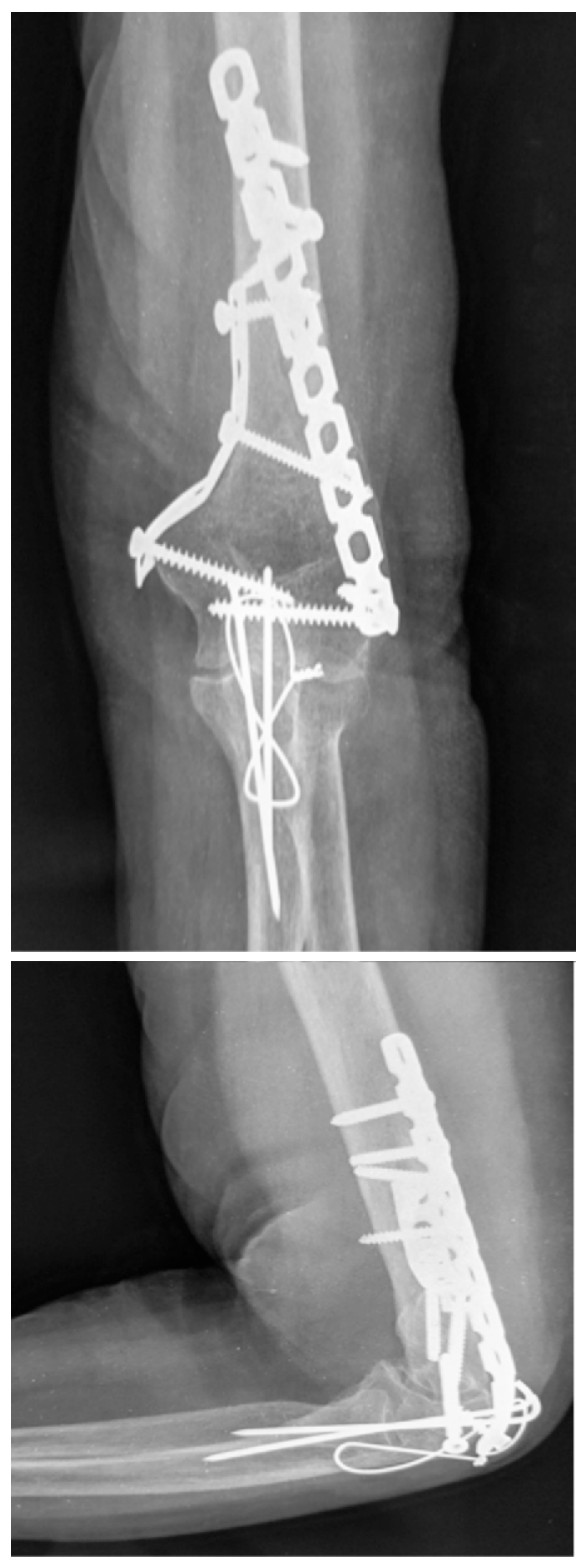

Figure.2: Post-operative imaging
Postoperatively all patients underwent long arm plaster of Paris slab for two weeks. All wounds healed primarily with no wound dehiscence. After two weeks sutures were removed and after one month of surgery slab were removed and patients were put on physiotherapy. Patients were followed up for 1-3 months and the results were assessed at twelve months using Mayo elbow Performance score system.

All the relevant information was entered into Microsoft word, statistical package for social services (SPSS) version 20, Microsoft excel and analyzed.

\section{RESULT}

There were total 16 patients, 10 male and 6 female ranging from 24 to 48 years enrolled in the study with an average mean age of 33.37 years \pm SD 8.30.The mechanism of injury included fall from tree in 6 patients, fall from cliff in 3 patients and one patient was in fall from low level ground, all together the commonest cause of fracture $(62.5 \%)$, followed by road traffic accidents (RTA) $37.5 \%$.All of them were operated within first week of injury. The average hospital stay was 10.69 days after surgery. All 16 patients were reviewed at a mean follow up of 9.38 months (range 6-12 months) and were evaluated clinically and radiologically for one year at 1, 3, 6, 9 and 12 months. Maximum number of fracture union was achieved by 16 weeks with mean 33.93 days \pm SD 4.58 .

$25 \%$ of patients had range of motion $10-120$ degree. $50.1 \%$ of patients had range of motion in between 120-130 degree. Only one patient achieved maximum range of motion up 140 degree. Two of five patients with $13 \mathrm{C} 3$ fractures and two of six patients with $13 \mathrm{C} 2$ fracture had flexion deformity of less than 30 degree. Three patients had hardware prominence which was addressed by removing the tension band wiring at 6 months once union was achieved. The arc of motion (range 60130 )with mean arc of motion was 100.31 SD \pm 20.85 degrees. The functional outcome based on Mayo elbow performance scoring system (MEPS) had excellent result in $8(50 \%)$ patients whereas each $4(25 \%)$ had good and fair results respectively.

Among eight excellent outcome, four, three and one of them were belonged to $13 \mathrm{C} 1,13 \mathrm{C} 2$ and $13 \mathrm{C} 3 \mathrm{AO}$ fracture type respectively which was found to be statistically insignificant association $(P=0.363)$. Meanwhile, pre and post up

\begin{tabular}{|c|c|c|c|c|c|}
\hline \multirow{2}{*}{ MEPS } & \multicolumn{3}{|c|}{ AO classification } & \multirow{2}{*}{ Total } & \multirow{2}{*}{$p$ value } \\
\hline & $13 C 1$ & $13 C 2$ & $13 C 3$ & & \\
\hline Excellent (>90) & $4(80 \%)$ & $3(50 \%)$ & $1(20 \%)$ & $8(50 \%)$ & \multirow{4}{*}{0.363} \\
\hline Good (75-89) & $0(0 \%)$ & $2(33.3 \%)$ & $2(40 \%)$ & $4(25 \%)$ & \\
\hline Fair (60-74) & $1(20 \%)$ & $1(16.7 \%)$ & $2(40 \%)$ & $4(25 \%)$ & \\
\hline Poor $(<60)$ & $0(0 \%)$ & $0(0 \%)$ & $0(0 \%)$ & $0(0 \%)$ & \\
\hline
\end{tabular}

Distribution of Mayo Elbow Performance Scoring System (MEPS) according to AO classification $(N=16)$ 
complication had equal chance of having statistically significant association with this fracture and surgical procedure. $(P=$ 0.002 , i.e.<0.05)

\section{DISCUSSION}

Intercondylar fracture of distal humerus in adults are difficult to manage due to complex anatomy of the elbow, small sized fracture fragments and the limited amount of subchondralbone. ${ }^{6}$ Surgical management is the treatment of choice. The correct method of using accurate size and number of plates and screw can prevent complications like non-union, stiffness and pain. The main goals of operative treatment are restoration of joint anatomy and stable fragment fixation. After establishment of complete union of the fracture, early initiation of physical therapy is the pre requisite for regaining a functional activity ${ }^{7,8,9}$.

Recently Kumar et al. showed that it is possible to obtain excellent outcome in distal one third of fracture using only single 4.5-mm LCP with two screw (4 cortices) keep in distal third fragment ${ }^{10}$. Atalar et al. used parallel plating in 21 communited distal humerus fractures. All fractures united but there were heterotrophic ossification in 33\% and chondrolysis in one patient. Parallel plating implies greater periosteal stripping and elevation of triceps and brachioradialis ${ }^{11}$. According to our experience it is mandatory to use two platesof $3.5 \mathrm{~mm}$ in a perpendicular fashion because clinical and biomechanical results have shown that double plate osteosynthesis is the most feasible and stable method of osteosynthesis ${ }^{12,13,14,15}$.

Sixty one patients with olecranon osteotomy for distal humerus fractures were followed up for six years by Coles CP et al. ${ }^{16}$ They observed that all osteotomies united and found that the problem with olecranon osteotomy seems to be the prominence of implants. In our study three patients had hardware prominence which was addressed by removing the tension band wiring at 6 months once union was achieved.

Young patient attained good movement at the elbow joint with active physiotherapy. As age increases the range of motion decreases. In present study, $25 \%$ of patient had range of
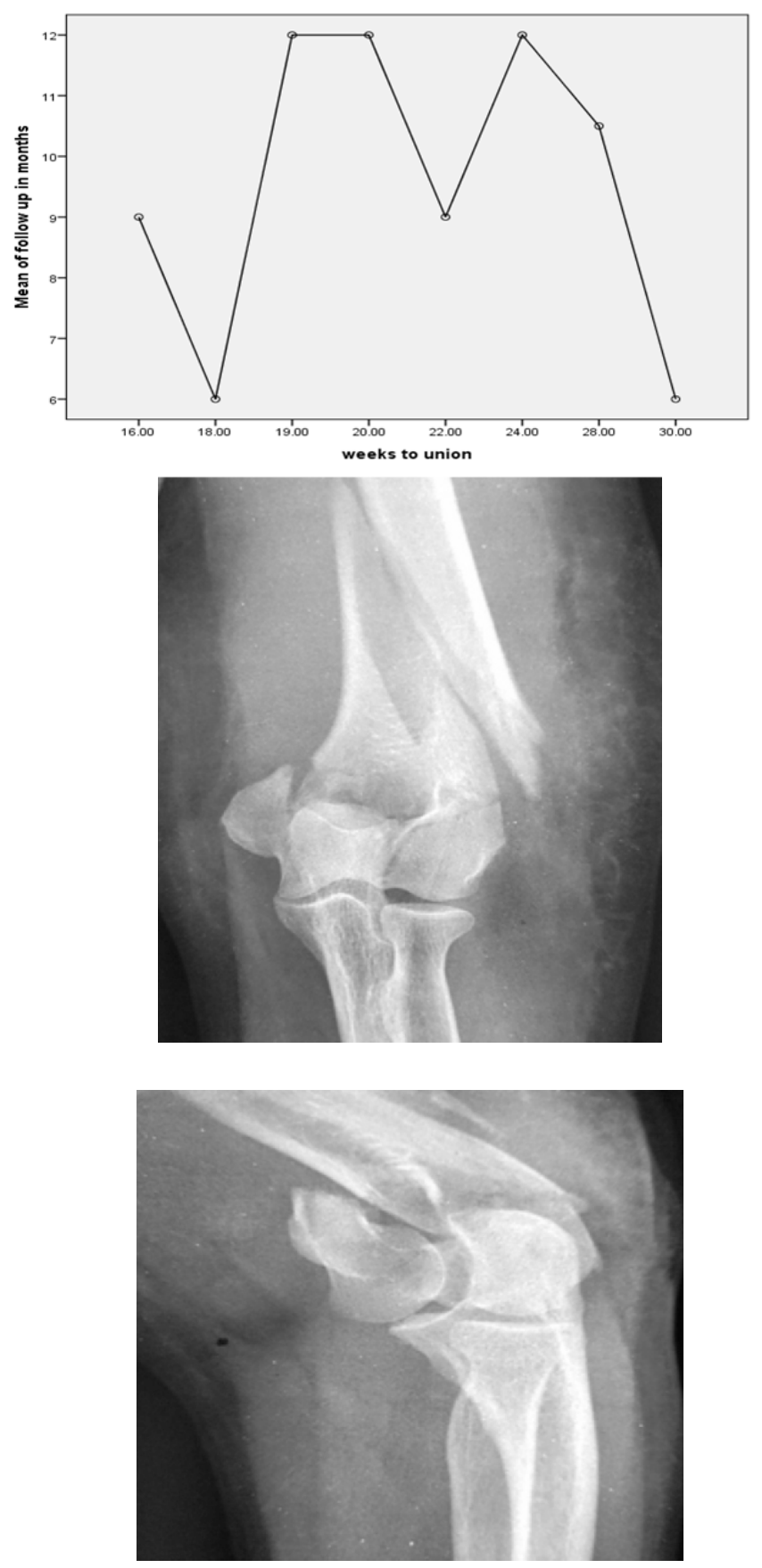

Figure 1: Pre-operative imaging

\begin{tabular}{|l|c|c|c|c|}
\hline \multicolumn{5}{|c|}{ AO classification } \\
\hline Variables & C1 & C2 & C3 & P value \\
\hline No. of patients & 5 & 6 & 5 & 0.93 \\
\hline Average Weeks to union & $21.4 \pm 4.4$ & $20.6 \pm 4.1$ & $20.8 \pm 6$ & 0.4 \\
\hline Average Range of motion & $124 \pm 11.4$ & $20 \pm 8.9$ & $108 \pm 8.3$ & 0.35 \\
\hline Average AOM & $106 \pm 27$ & $102.5 \pm 17.8$ & $92 \pm 19.2$ & 0.34 \\
\hline Average Follow up in months & $9.6 \pm 2.5$ & $9.5 \pm 2.2$ & $9 \pm 3$ & 0.84 \\
\hline Average Duration of hospital stay & $10.2 \pm 6$ & $11.5 \pm 6.6$ & $10.2 \pm 2.3$ & 0.13 \\
\hline
\end{tabular}

Data stratification according to AO classification to compare mean variation 
motion of 10-120 degree. Although age is not statistically relevant factor for functional activity, one patient had attained maximum range of motion $10-140$ degree at age of 44 years.

\section{Limitations of the study}

The sample size of the study and short follow up were the major limitation of the study which was conducted in a single hospital. Use of Computer tomography (CT) scan would have given exact and precise communition of fractures and its displacement. The study sample may not be representative of all the participants of similar age.

\section{CONCLUSION}

Dual 90-90 plating technique through Chevron olecranon osteotomy is also one of the good method for intercondylar reconstruction with rigid fixation like $A O 13 \mathrm{C}$ fractures, however controversies regarding positioning of plates still exists and need larger prospective trials with long duration of follow up.

\section{REFERENCES}

1. KuntzJr, David G, BaratzME.Fractures of the elbow. orthopclin North Am Jun.1999; 30(1):3761.

2. George S. Athwal Fractures of the distal humerus. Chapter-33 in Rockwood and Green's fractures in adults. $7^{\text {th }}$ Edn.Lippincott Williams and Wilkins.945-998.

3. Robinson CM, Hill R, Jacobs N, Dall G, Brown C. Adult humeral metaphyseal fractures. Epidemiology and results of treatment. J of orthop Trauma.2003:17: 38-47.

4. Nauth A, Mckee MD, Ristevski B, Hall J, Schemitch EJ. Current concepts review. Distalhumerus fractures in adults. J Bone joint surg (Am).2011;93:680-700.

5. Galano GJ, Ahmed AJ, Levine WN. Current treatment for bicolumnar distal humerus fractures. J Am Aced orthop surg. 2010; 18: 20-30.

6. Gupta R, Khanchandani P. intercondylar fracture of distal humerus in adults a critical analysis of 55 cases. Injury.2002;33(6):511-5.

7. Helfet DL, SchmelingGJ.Bicondylarintraarticular fractures of the distalhumerus in adults.Clinicalorthopaedics and related research.1993(292):26-36.

8. Henley MB, Bone LB, Parker B. Operative management of intraarticular fractures of the distal humerus.Journal of orthopaedic trauma.1987;1(1):24-35.

9. Self J, Viegas SF, Buford WL, Jr, Patterson RM. A comparison of double plate fixation methods for complex distal humerusfractures.Journal of shoulder and elbow surgery / Americal Shoulder and elbow Surgeons (et al).1995;4(1 Pt 1 ):10-6.

10. Kumar MN, Ravishankar MR, ManurR. Single locking compression plate fixation of extraarticular distal humeral fractures. JOrthopTraumatol 2015;16:99-104.

11. Atalar AC, Demirhan M, Salduz A, Kilicoglu O, Seyahi A. Functional results of the parallel plate technique for complex distal humerusfractures. ActaOrthopTraumatol Turc.2009;43(1):21-7.

12. Helfet $\mathrm{DL}$, Hotchkiss RN. Internal fixation of the distal humerus: a biomechanical comparision of methods. Journal of orthopaedic trauma. 1990:4(3):206-4.

13. Jupiter JB, Neff $U$, Holzach P, AllgowerM.Intercondylar fractures of the humerus.An operative approach.The journal of bone and joint surgery American volume.1985;67(2):226-39.

14. GuptaR.Intercondylar fractures of the distalhumerus in adults.Injury.1996;27(8):569-72.

15. Kinzl I, Fleischmann W. The treatment of distal upper arm fractures.Der Unfallchirurg.1991;94(9):455-460.

16. Coles CP, Barei DP, Nork SE, Taitsman LA, Hanel DP, Bradford Henley $M$. The olecranon osteotomy: a six year experience in the treatment of intraarticular fractures of the distal humerus. $J$ Orthop Trauma,2006;20(3):164-71. 